T cell subpopulations, and total and KLH-specific immunoglobulin (Ig) levels were monitored. Additionally, gene expression in blood was analysed using the NanoString nCounter platform.

Results: Atacicept was well-tolerated at all dose levels tested. All animals in the treatment arms had quantifiable levels of atacicept in serum throughout the study. Reduction of serum IgM and IgG was detected 7 days after treatment, with a continuous reduction in the mean serum $\lg M$ and $\lg$ G levels observed until Day 29 (table 1). In animals treated with 3 and $30 \mathrm{mg} / \mathrm{kg}$ atacicept, a significant decrease in serum anti-KLH lgG levels was observed versus vehicle-treated controls beginning at Day 11 (table 2). A minor reduction in absolute CD3-CD20+, IgD +B cell numbers was seen in response to treatment, but no changes in $T$ cell subsets were detected. Changes in gene expression following atacicept treatment were predominantly observed in B cell-related and Ig genes.

Abstract FRI0272 - Table 1. Mean\% reduction of total IgM and IgG relative to baseline

\begin{tabular}{lcccccc}
\hline Mean reduction & \multicolumn{3}{c}{ Day 8} & \multicolumn{3}{c}{ Day 29} \\
\cline { 2 - 7 } & $0.3 \mathrm{mg} / \mathrm{kg}$ & $3 \mathrm{mg} / \mathrm{kg}$ & $30 \mathrm{mg} / \mathrm{kg}$ & $0.3 \mathrm{mg} / \mathrm{kg}$ & $3 \mathrm{mg} / \mathrm{kg}$ & $30 \mathrm{mg} / \mathrm{kg}$ \\
\hline IgG & 7.1 & 14.2 & 16.3 & 10.6 & 27.2 & 46.3 \\
IgM & 9.1 & 17.1 & 22.3 & 21.5 & 52.3 & 64.1 \\
Table 2 Mean\% reduction of KLH-specific IgM and IgG relative to vehicle group \\
Mean reduction \\
\multicolumn{7}{c}{ Day 11} \\
KLH-specific IgG & $0.3 \mathrm{mg} / \mathrm{kg}$ & $3 \mathrm{mg} / \mathrm{kg}$ & $30 \mathrm{mg} / \mathrm{kg}$ & $0.3 \mathrm{mg} / \mathrm{kg}$ & $3 \mathrm{mg} / \mathrm{kg}$ & $30 \mathrm{mg} / \mathrm{kg}$ \\
KLH-specific IgM & 17.3 & 55.2 & 49.7 & 39.4 & 78.8 & 71.5 \\
\hline
\end{tabular}

Conclusions: This study showed that atacicept modulates B cell responses, IgM and IgG levels, and Ig isotype switching in a KLH-antigen-challenged cynomolgus monkey model, thus supporting its use in the treatment of antibody-mediated diseases. Additionally, gene expression PD markers identified in this study will be used in subsequent clinical trials as exploratory PD readouts.

Disclosure of Interest: E. Samy Employee of: EMD Serono Research and Development Institute, Inc. (a business of Merck KGaA, Darmstadt, Germany), A. Bender Employee of: EMD Serono Research and Development Institute, Inc. (a business of Merck KGaA, Darmstadt, Germany), Y. Wu Employee of: EMD Serono Research and Development Institute, Inc. (a business of Merck KGaA, Darmstadt, Germany), V. Castagna Employee of: Istituto di Ricerche Biomediche Antoine Marxer RBM SpA, an affiliate of Merck KGaA, E. Bertotti Employee of: Istituto di Ricerche Biomediche Antoine Marxer RBM SpA, an affiliate of Merck KGaA, R. Boggio Employee of: Istituto di Ricerche Biomediche Antoine Marxer RBM SpA, an affiliate of Merck KGaA, A. Paoletti Employee of: Istituto di Ricerche Biomediche Antoine Marxer RBM SpA, an affiliate of Merck KGaA, S. Riva Employee of: Istituto di Ricerche Biomediche Antoine Marxer RBM SpA, an affiliate of Merck KGaA, P. Schneider Grant/research support from: EMD Serono Research and Development Institute, Inc. and Merck KGaA, P. Haselmayer Employee of: Merck KGaA, J. DeMartino Employee of: EMD Serono Research and Development Institute, Inc. (a business of Merck KGaA, Darmstadt, Germany)

DOI: 10.1136/annrheumdis-2018-eular.3712

\section{FRI0273 RNA SEQUENCING DETECTION OF GENE DYSREGULATION IN EPITHELIAL SORTED CELLS FROM SALIVARY GLAND TISSUE REVEALS INTERESTING PATHWAYS INVOLVED IN SJÖGREN'S SYNDROME PATHOPHYSIOLOGY}

E. Rivière ${ }^{1}$, N. Tchitchek ${ }^{1}$, G. Nocturne ${ }^{1,2}$, S. Boudaoud ${ }^{1}$, J. Pascaud $^{1}$, A. Virone $^{1}$, A. Thai ${ }^{3}$, N. Allaire ${ }^{3}$, B. Jagla ${ }^{4}$, M. Mingueneau ${ }^{3}$, X. Mariette ${ }^{1,2} .{ }^{1} /$ mmunology of viral infections and autoimmune diseases, IDMIT, CEA - Université Paris SudINSERM U1184, Le Kremlin Bicêtre and Fontenay aux Roses; ${ }^{2}$ Rheumatology, Université Paris Sud, Le Kremlin Bicêtre, France; ${ }^{3}$ Immunology Research, Biogen, Cambridge, USA; ${ }^{4}$ Biomarker Discovery Platform UtechS CB and Hub de Bioinformatique et Biostatistiques C3BI, Institut Pasteur, Paris, France

Background: Primary Sjögren's syndrome (pSS) is a chronic autoimmune disorder characterised by lymphocytic infiltrates and destruction of the salivary glands. Several lines of evidence support the hypothesis that salivary gland epithelial cells (SGECs) are not only the target of autoimmunity in pSS patients but may also play a role for its initiation and maintenance.

Objectives: To establish high-resolution molecular maps of SGEC from pSS patients compared to controls using RNASeq analysis.

Methods: Patients had pSS according to 2016 EULAR/ACR criteria and controls had sicca symptoms without any antibodies and with normal lip biopsy. SGEC, B, T CD4 and CD8 lymphocytes were sorted from salivary gland biopsies from 9 pSS patients and 4 controls, using a FACS ARIA cell sorter. Total RNASeq profiling was performed using MiSeq (Illumina). For SGEC subset, 4 samples were excluded due to a contamination by $\mathrm{B}$ lymphocytes, thus analysis was performed on 5 pSS and 4 controls using R software, to identify transcriptional differences between pSS and control SGEC. Functional Enrichment analysis was performed using Ingenuity Pathway Analysis software.

Results: In SGEC, 495 genes were differentially expressed between pSS and controls. 280 genes were up-regulated, and 215 genes were down-regulated Enrichment analysis (table 1) highlighted IL-7 signalling pathways (including IL-7, STAT5A, STAT1 genes) and interferon signalling (including OAS1, IFIT3, IFI6, TAP1 genes). Other genes potentially involved in immune responses and interactions between SGEC and lymphocytes were significantly up-regulated, including bone marrow stromal cell antigen 2, HLA-DRA, BAFF-R and IL-23 A (table 2). These results need to be confirmed by RT qPCR. However, consistent results have already been obtained in our laboratory, showing that IL-7 serum level is increased in pSS patients compared to controls and that SGECs produce IL-7 after interferon stimulation. The analysis of the non-coding RNA part and the other sorted cells subtypes is ongoing

Abstract FRI0273 - Table 1. Pathways identified as statistically over-represented with Ingenuity Pathways Analysis in epithelial cells from pSS patients compared to controls

\section{Pathway} -log p-value

Primary Immunodeficiency Signalling 4,08

Interferon Signalling

B Cell Development

Role of JAK2 in Hormone-like Cytokine Signalling

2,89

IL-7 Signalling Pathway

Abstract FRI0273 - Table 2. Selection of genes differentially expressed between pSS and controls in SGEC

\begin{tabular}{lcc}
\hline Gene Symbol & log2 fold-change & p-value \\
\hline IL-7 & 2.56 & 0.002 \\
BST2 & 4.08 & 0.0002 \\
HLA-DRA & 1.84 & 0.0372 \\
IL-23 A & 3.59 & 0.0155 \\
BAFF-R & 4.94 & 0.0097 \\
\hline
\end{tabular}

Conclusions: Immune interactions between SGEC and B or T lymphocytes could represent a key in the understanding of the initiation and/or maintenance of autoimmunity in pSS. Our study highlights the key role of epithelial cells in activation of immune cells. In vitro experiments are needed to confirm these results and elucidate the molecular mechanisms.

Acknowledgements: Arthritis Fondation Courtin, Arthritis R and D for providing a PhD fellowship

Disclosure of Interest: None declared

DOI: 10.1136/annrheumdis-2018-eular.3307

\section{FRI0274 ELEVATED REACTIVITY OF CD38HIGHIGD+ B CELLS AGAINST BAFF CONTRIBUTES TO IGG OVERPRODUCTION IN PATIENTS WITH PRIMARY SJÖGREN'S SYNDROME}

E. Ishioka-Takei, K. Yoshimoto, K. Suzuki, H. Yasuoka, K. Yamaoka, T. Takeuchi. Keio University School of Medicine, Tokyo, Japan

Background: Primary Sjögren's syndrome (pSS) is often accompanied by hypergammaglobulinemia and production of autoantibodies, such as anti-Ro/SSA and anti-La/SSB antibodies. These serological aberrations suggest that abnormally activated $B$ cells play a key role in the pathogenesis of pSS. We have previously reported that the proportion of peripheral $C D 38^{\text {high }} / g D^{+} B$ cells among $C D 19^{+} B$ cells is significantly elevated in pSS patients and positively correlated with serum anti-Ro/SSA, anti-La/SSB titer, total IgG, and the European League against Rheumatism (EULAR) Primary Sjögren's syndrome disease activity index (ESSDAI). B cell activating factor belonging to TNF family (BAFF) is a well known cytokine which promotes differentiation, proliferation and survival of $B$ cells. It has been reported that serum BAFF level is increased in pSS patients compared to $\mathrm{HC}$ and that BAFF is also highly expressed in salivary glands. Based on these back ground, it is conceivable that BAFF plays a pivotal role in the pathogenesis of pSS.

Objectives: To elucidate the involvement of BAFF in IgG overproduction in pSS. Methods: Peripheral CD19+ B cells were prepared from pSS patients $(n=16)$ and gender-matched $\mathrm{HC}(\mathrm{n}=10)$ by using CD19-microbeads. The cells were stimulated in vitro with an anti-IgM antibody, recombinant human CD40 ligand and recombinant human IL-4 ('multiple stimulation') with or without recombinan human soluble BAFF (rhsBAFF) for 96 hours. The amount of IgG produced by the cells in the culture supernatants was measured by ELISA. The proportion of $B$ cell subsets, characterised by anti-CD19, anti-lgD and anti-CD38 antibodies, and the expression level of a BAFF receptor (BR3) in the subsets were analysed by FACS. Disease activities of the pSS patients were quantified based on the ESSDAI scores. The serological data of the patients were collected by clinical records. 